\title{
Multi-thermal dynamics and energetics of a coronal mass ejection in the low solar atmosphere ${ }^{\star}$
}

\author{
I. G. Hannah and E. P. Kontar
}

\begin{abstract}
SUPA School of Physics \& Astronomy, University of Glasgow, Glasgow, G12 8QQ, UK
e-mail: iain.hannah@glasgow.ac.uk
\end{abstract}

Received 31 May 2012 / Accepted 30 January 2013

\begin{abstract}
Aims. The aim of this work is to determine the multi-thermal characteristics and plasma energetics of an eruptive plasmoid and occulted flare observed by the Solar Dynamics Observatory's Atmospheric Imaging Assembly (SDO/AIA).

Methods. We study a 2010 Nov. 3 event (peaking at 12:20 UT in GOES soft X-rays) of a coronal mass ejection and occulted flare that demonstrates the morphology of a classic erupting flux rope. The high spatial and time resolution and six coronal channels of the SDO/AIA images allows the dynamics of the multi-thermal emission during the initial phases of eruption to be studied in detail. The differential emission measure is calculated, using an optimized version of a regularized inversion method, for each pixel across the six channels at different times, resulting in emission measure maps and movies in a variety of temperature ranges.

Results. We find that the core of the erupting plasmoid is hot $(8-11,11-14 \mathrm{MK})$ with a similarly hot filamentary "stem" structure connecting it to the lower atmosphere, which could be interpreted as the current sheet in the flux rope model, though is wider than these models suggest. The velocity of the leading edge of the eruption is 597-664 $\mathrm{km} \mathrm{s}^{-1}$ in the temperature range $\geq 3-4 \mathrm{MK}$ and between $1029-1246 \mathrm{~km} \mathrm{~s}^{-1}$ for $\leq 2-3 \mathrm{MK}$. We estimate the density (in 11-14 MK) of the erupting core and stem during the impulsive phase to be about $3 \times 10^{9} \mathrm{~cm}^{-3}, 6 \times 10^{9} \mathrm{~cm}^{-3}, 9 \times 10^{8} \mathrm{~cm}^{-3}$ in the plasmoid core, stem, and surrounding envelope of material. This gives thermal energy estimates of $5 \times 10^{29} \mathrm{erg}, 1 \times 10^{29} \mathrm{erg}$, and $2 \times 10^{30} \mathrm{erg}$. The kinetic energy for the core and envelope is slightly lower. The thermal energy of the core and current sheet grows during the eruption, suggesting continuous influx of energy presumably via reconnection.

Conclusions. The combination of the optimized regularized inversion method and SDO/AIA data allows the multi-thermal characteristics (i.e. velocity, density, and thermal energies) of the plasmoid eruption to be determined.
\end{abstract}

Key words. Sun: X-rays, gamma rays - Sun: UV radiation - Sun: activity - Sun: flares - Sun: corona - techniques: image processing

\section{Introduction}

The solar atmosphere is awash with highly dynamic phenomena driven by the rapid liberation of magnetically stored energy. Magnetic reconnection is thought to facilitate this energy release, resulting in solar flares, coronal mass ejections (CMEs), and the associated particle acceleration, plasma heating, and bulk motions. The classic model of solar eruptive events (SEEs) is the CSHKP model (Carmichael 1964; Sturrock 1966; Hirayama 1974; Kopp \& Pneuman 1976), which continues to become more ornate over time (for instance Lin \& Forbes 2000). The basic scenario is that of an erupting flux rope (helical magnetic structure) that stretches and elongates the coronal magnetic field behind it, instigating magnetic reconnection in a vertical current sheet. This releases considerable energy, particularly in accelerated particles that then travel down into the denser lower solar atmosphere. Here they lose their energy through collisions with the background plasma, producing hard $\mathrm{X}$-ray (HXR) footpoints and flare ribbons, heating material that then expands upwards filling flaring loops.

Flares are clearly observed in the lower solar atmosphere, but CMEs have, until recently, mostly been studied at higher altitudes (for an overview see Hudson et al. 2006; Webb \& Howard

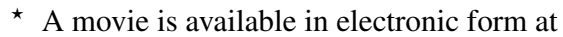
http: //wWw . aanda.org
2012) owing to the lack of suitable high cadence observations. This means that there is considerable uncertainty as to the specific processes that develop flux ropes and initiate CMEs (e.g. Démoulin \& Aulanier 2010). High in the corona, white-light observations typically show CMEs structured as a bright loop ahead of a darker cavity containing a bright core. Here the bright loop is taken to be due to the increased density of material amassed in the leading front of the eruption with the cavity likely due to a flux rope (Gibson et al. 2006). CMEs have been found to evolve in distinct stages, initiation, acceleration, and propagation (Zhang \& Dere 2006), and the initial acceleration stage of CME synchronizes well with the flare HXR (Kahler et al. 1988; Sterling \& Moore 2005; Temmer et al. 2008, 2010) and soft X-ray (SXR) emission (Zhang et al. 2001; Vršnak et al. 2005), and the HXR flux correlates with the acceleration of CMEs (Temmer et al. 2010). Active regions are significantly more likely to be eruptive if they have a sigmoidal morphologly (Canfield et al. 1999), and these structures are seen as the precursors to flux ropes both in observations (Liu et al. 2010; Green et al. 2011) and in simulations (Török \& Kliem 2003; Fan \& Gibson 2003; Archontis \& Török 2008; Aulanier et al. 2010).

Associated with CMEs is the nearby dimming of the corona during the onset stage, seen in SXRs (Sterling \& Hudson 1997) and EUV (e.g. Gopalswamy \& Hanaoka 1998). This could be due to the depletion of material as part of the eruption, plasma heating, or a combination of both. Observations generally point 


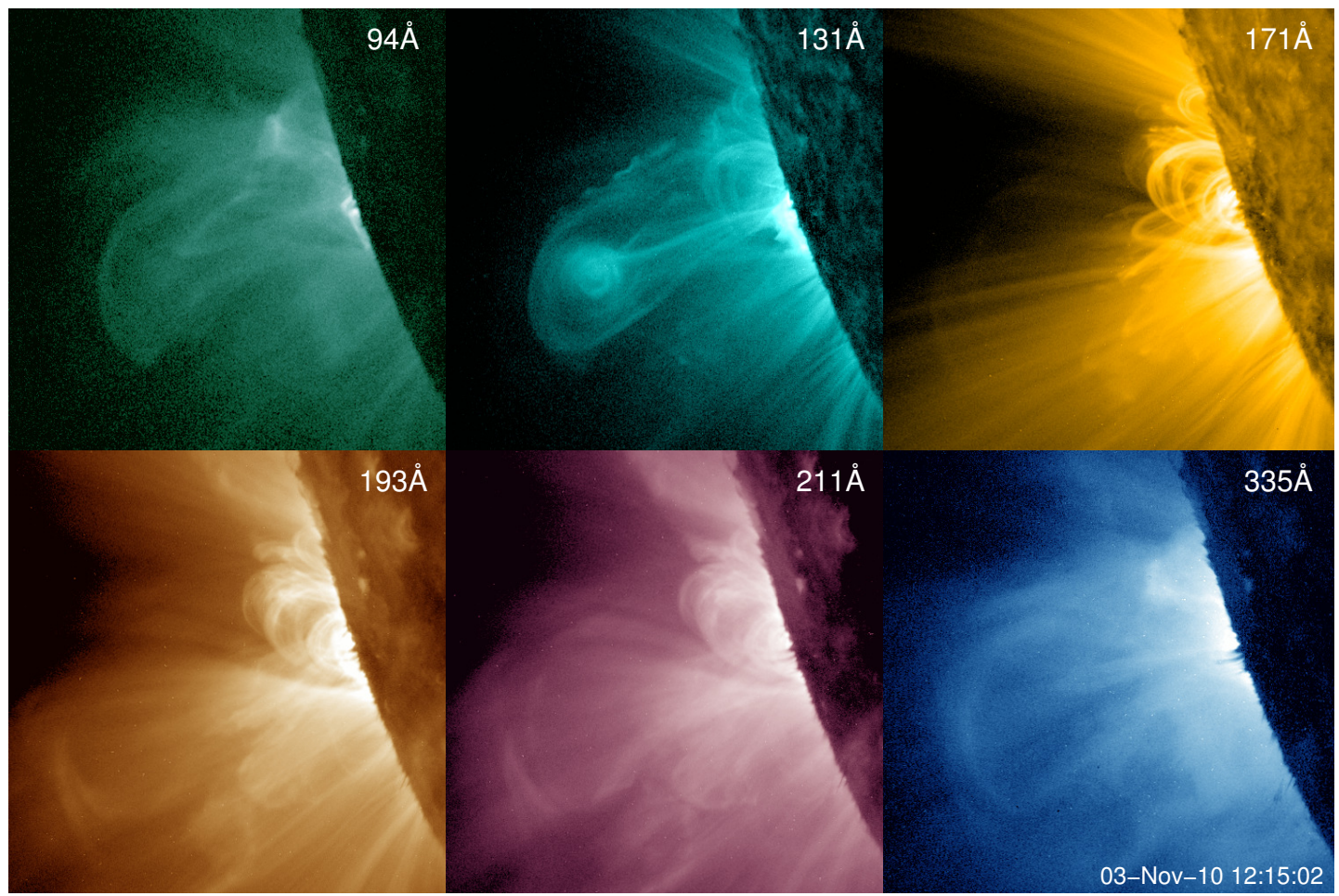

Fig. 1. SDO/AIA observations from each of the six coronal filters during the impulsive phase of the 3-Nov-2010 flare when the erupting plasmoid is clearly visible in $131 \AA$. The time shown is for the $94 \AA$ image, with all from 12:15:00 to 12:15:09 UT.

to the former; for instance, five CMEs studied by Harrison et al. (2003) using EUV spectroscopic data from SOHO/CDS showed dimming from mass loss and not temperature variations. However, there are also examples of CMEs where the dimming might be due to plasma heating and not depletion (Robbrecht \& Wang 2010).

Spectral observations of CMEs in the extended corona ( $>1.5 R_{\odot}$ from Sun centre) with $\mathrm{SOHO} / \mathrm{UVCS}$ revealed many aspects of CMEs at these altitudes (Kohl et al. 2006), observing spectral lines over 0.02-6 MK. In one CME a "narrow" (100 Mm) structure was observed for $20 \mathrm{~h}$, with an emission measure of about $10^{25} \mathrm{~cm}^{-5}$ over $4-6 \mathrm{MK}$ connecting the CME core to the flare loops below (Ciaravella et al. 2002). This was interpreted as an edge-on view of the current sheet, and a density of $5 \times 10^{7} \mathrm{~cm}^{-3}$ was estimated using a $40 \mathrm{Mm}$ line-of-sight component. Similarly hot current sheets have been observed in another long-duration event (Ko et al. 2003), as have as faster ones (Lin et al. 2005) where the leading edge and CME core were moving at 1939 and $1484 \mathrm{~km} \mathrm{~s}^{-1}$ respectively. Hotter current sheets have been observed in some CMEs as well (Innes et al. 2003; Bemporad et al. 2006; Schettino et al. 2010). Landi et al. (2010) used Hinode/EIS to determine the differential emission measure (DEM, see Sect. 2) of a CME lower in the solar atmosphere (at $\left.1.1 R_{\odot}\right)$. They find a multiple-peaked DEM, with one component at $0.13 \mathrm{MK}$ of density $10^{10-11} \mathrm{~cm}^{-3}$ and another at $0.5 \mathrm{MK}$ of density $10^{8.7} \mathrm{~cm}^{-3}$ travelling together. The emission at about $1 \mathrm{MK}$ came from the line-of-sight/background corona; however, SXR emission was also detected by Hinode/XRT, enveloping the core and suggesting material at 5-10 MK. The analysis of the post eruption arcade found similar plasma to the current sheet, both coronal plasma heated up to $3 \mathrm{MK}$ (Landi et al. 2012b)

High spatial $\left(\sim 1.5^{\prime \prime}\right)$ and temporal resolution $(\sim 12 \mathrm{~s})$, EUV full disk images in multiple wavelengths is now available from the Atmospheric Imaging Assembly (Lemen et al. 2012) on the Solar Dynamics Observatory (SDO/AIA), with several authors taking advantage of this data to study CME formation and propagation from the low corona (e.g. Patsourakos et al. 2010; Kozarev et al. 2011). One particular event, which peaked in GOES SXRs at 12:20 UT 2010-Nov-03 (Fig. 1), an occulted flare and erupting plasmoid, unambiguously shows the formation of a flux rope and has subsequently been studied in detail (Reeves \& Golub 2011; Cheng et al. 2011; Foullon et al. 2011; Bain et al. 2012; Savage et al. 2012; Cheng et al. 2012). It is studied further in this paper. This event shows the formation of a flux rope clearly in the $131 \AA$ images, with a peak in their sensitivity at $11 \mathrm{MK}$, though dimming at the core in channels sensitive to cooler emission ( $171 \AA, 211 \AA$ ), shown in Fig. 1 . As the plasmoid move outwards, a filamentary, stem-like structure forms behind it, again sharply defined in $131 \AA$ and suggestive of a large-scale current sheet (Reeves \& Golub 2011; Cheng et al. 2011; Savage et al. 2012). The kinematics of the eruption in the different channels has been found to be slower (about $600 \mathrm{~km} \mathrm{~s}^{-1}$ versus $1400 \mathrm{~km} \mathrm{~s}^{-1}$ ) in those channels sensitive to hotter emission (Cheng et al. 2011; Bain et al. 2012).

The propagation of a metric Type II radio burst ahead of the CME in EUV is faster still (nearing $2000 \mathrm{~km} \mathrm{~s}^{-1}$ ), suggesting a piston-drive shock, in which it is moving too quickly for the ambient plasma to flow behind the driver (Bain et al. 2012). This work also found that HXR emission observed with RHESSI had two non-thermal (18-40 keV) sources, one compact low in the corona, the other extended and high in the corona and close to the erupting plasmoid. The latter could be due to electrons accelerated in the stem/current sheet. Although this event superficially matches the cartoon model of flux rope eruption, things are more complicated: Foullon et al. (2011) find Kelvin-Helmholtz waves along the top edge of the erupting plasma, possibly allowing for 
a secondary reconnection process. Savage et al. (2012) studied the inflow and outflow into the CS region, finding the hottest material flowing in fastest and correlating with the RHESSI thermal $\mathrm{X}$-ray emission. Pairs of inflowing and outflowing material were found to be travelling away from the region faster than they arrived, suggesting acceleration.

Since SDO/AIA provides six different wavelength channels, which are sensitive to a range of coronal temperatures, they can be used to determine the DEM of the different parts of the CME. Cheng et al. (2012) did this for this event, as well as two others, producing DEMs for different CME regions: the core of the erupting flux rope and the leading front. They found the cores to have emission over a broad distribution of temperatures $(3 \leq T \leq 20 \mathrm{MK})$, with an average temperature of about $8 \mathrm{MK}$ and densities of about $10^{9} \mathrm{~cm}^{-3}$. The leading front of the eruptions were cooler (about $2 \mathrm{MK}$ on average) with a narrower temperature distribution $(1 \leq T \leq 3 \mathrm{MK})$ similar to the preeruption coronal material but with slightly higher densities. They interpreted the leading fronts to be the signatures of compressed ambient coronal plasma. Dimming, about ten minutes after the eruption, was found to be due to the rarefaction of material.

In this paper we use a different approach, regularized inversion, to produce the DEM not for a few regions within the CME but for every single pixel in the set of six SDO/AIA images, every $12 \mathrm{~s}$ throughout the event, thus allowing the highly dynamic multi-thermal properties of the CME to be investigated. The regularized approach used in this paper (see Sect. 2) is an optimized version of our original code, which was introduced, tested, and compared to the approach used by Cheng et al. (2012) in Hannah $\&$ Kontar (2012). The version presented here is able to compute $10000 \mathrm{~s}$ of DEMs per second, as well as calculating both horizontal and vertical uncertainties (see Sect. 2.2), and without forcing a prescribed DEM model. In Sect. 3.2 the properties of the DEM in different pixels of the event are discussed, with the maps and their uncertainties presented in Sect. 3.3. We then investigate the time evolution of the DEMs in terms of the morphology, total emission, and propagation speed for the different temperature ranges, in Sect. 3.4. Since the erupting plasmoid and filamentary stem structure is well defined at high temperatures (11-14 MK), we use the emission measures in Sect. 3.5 to estimate the densities and thermal energy of the different components.

\section{Differential emission measures}

\subsection{Introduction}

The signal detected $g(x, y, t, i)$ in each pixel $(x, y)$ in the $i$ th SDO/AIA filter at time $t$ can be interpreted as

$g(x, y, t, i)=\int_{T} K(T, i) \xi(T, x, y, t) \mathrm{d} T+\delta g(x, y, t, i)$,

where $K(T, i)$ is the temperature response of the $i$ th filter (O'Dwyer et al. 2010; Boerner et al. 2012). The DEM $\xi(T, x, y, t)$, at a particular instance in one pixel, is taken to be $\xi(T)=n(h)^{2} \mathrm{~d} h / \mathrm{d} T\left[\mathrm{~cm}^{-5} \mathrm{~K}^{-1}\right]$, which is the electron density $n^{2}$ along the line-of-sight $h$ of the emitting material at temperature $T$. The inherent noise in the observations $\delta g(x, y, t, i)$ is due to counting statistics, background, and instrumental uncertainties, will be significantly amplified when a direct solution of Eq. (1) is attempted. This recovery of the DEM is an ill-posed inverse problem (Tikhonov 1963; Bertero et al. 1985; Craig \& Brown 1986; Prato et al. 2006) with the growth of uncertainties resulting in spurious solutions. To recover useful solutions to the problem given in Eq. (1), it needs to be constrained to avoid the noise amplification in the DEM solution. The constraints are often physically motivated additional information a priori known about the solution. An additional challenge here is that we need an approach that can quickly and robustly calculate DEMs since there is a wealth of SDO/AIA data for studying this highly dynamic eruption. The simple isothermal/ratio approach (e.g. Vaiana et al. 1973) places a very strict, though possibly erroneous, constraint on the DEM solutions and so it is ill-suited to this problem.

An alternative is to forward fit a chosen DEM model to the data, for example with multi-Gaussians (e.g. Aschwanden \& Boerner 2011) or splines (e.g. Weber et al. 2004), the latter used for this CME event by Cheng et al. (2012). Again, if the assumption about the form of the DEM is wrong, then the results are difficult to interpret reliably. These techniques can be computationally slow, only allowing select regions to be analysed, and not taking full advantage of the SDO/AIA data. Other approaches, such as MCMC (Kashyap \& Drake 1998), can produce very robust results, without requiring predefined functional forms to the DEM solutions, and uncertainty estimates, but are computationally slow and so are again inappropriate for obtaining DEM maps.

Tikhonov regularized inversion recovers the solution by limiting the amplification of the uncertainties through assumptions about its "smoothness" by using linear constraints (Tikhonov 1963; Craig 1977; Prato et al. 2006). This approach does not make any assumption on the functional form of DEM, and we have implemented an algorithm (Hannah \& Kontar 2012) to recover the DEM from solar data using Tikhonov regularization and generalized singular value decomposition GSVD (Hansen 1992). This code, originally developed and extensively tested for the inversion of X-ray data (Kontar et al. 2004, 2005), naturally provides estimates to the uncertainties in the solution, an important feature when trying to recover a large number of DEMs. Recently, a simpler and computationally fast (computing $100 \mathrm{~s}$ to $1000 \mathrm{~s}$ DEMs per second) SVD regularization approach has been applied to AIA data (Plowman et al. 2012), however it does not produce error estimates. Regularization has also been used with solar data to determine the 3D emission measure through the use of solar rotation or multi-spacecraft observations (Frazin et al. 2005, 2009). In general, regularized approaches do not require a positive solution but one can be obtained by using an iterative method such a reiterated conjugate gradients, which was used for solar data by Fludra \& Sylwester (1986). Our approach uses one iteration to help with the weighting and positivity of the final solution, whilst maintaining the fast computational speed.

\subsection{Regularization method for DEM maps}

The full details of the method are in Hannah \& Kontar (2012), and this previous version of the algorithm allows a variety of different controls on the nature of the regularization but is too computationally slow to practically produce DEM maps and movies (running at about a few DEMs per second). We have produced an optimized and simplified version of the software ${ }^{1}$, explained below with considerable speed gains. Since the DEM calculation per pixel location is independent of other pixels in the same image, further speed gains were achieved by parallization of the code within IDL using Bridges. The results is that we can achieve about $1000 \mathrm{~s}$ DEMs per second on a standard multi-core

\footnotetext{
Available online:
}

http://www . astro.gla.ac.uk/ iain/demreg/map/ 

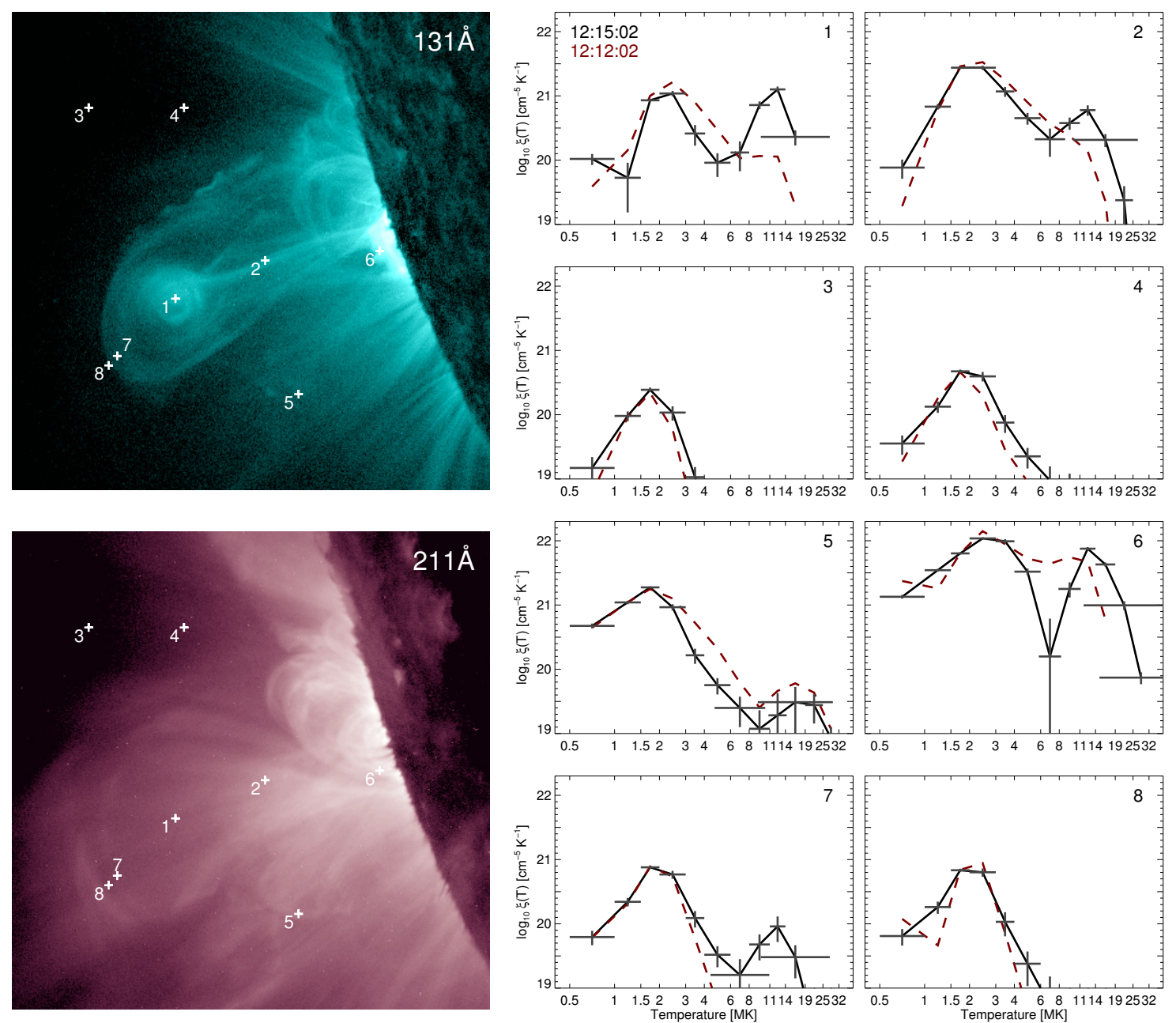

Fig. 2. Left: SDO/AIA $131 \AA$ and $211 \AA$ images from Fig. 1 with eight pixels highlighted. Right: the corresponding regularized DEM solutions $\xi(T)$ (using twelve temperature bins) and the vertical $\Delta \xi(T)$ and horizontal $\Delta \log T$ errors (the maximum of the error from the resolution matrix or the temperature bin width) for the highlighted pixels using the data from all six image in Fig. 1. The dashed lines show the DEM solutions in those pixels before the eruption at 12:12:02 UT, whereas the solid lines are from 12:15:02 UT.

cpu laptop/desktop and 10000 s DEMs per second on multi-cpu machines.

To obtain the DEM maps, we performed regularization with the zeroth-order constraint and no initial guess solution. For the detailed explanation of these parameters and the effect they have on the regularized solution, see Hannah \& Kontar (2012). The specific problem to be solved here is

$\|\widetilde{\mathbf{K}} \xi-\widetilde{\mathbf{g}}\|^{2}+\lambda\|\mathbf{L} \xi\|^{2}=\min$,

where the constraint matrix $\mathbf{L}$ is the identity matrix (normalized by the temperature bin width) and the tilde indicates normalization by the error in the data $\delta g$. The GSVD singular values $\left(\gamma_{i}\right.$, $\left.\beta_{i}\right)$ and vectors $(\boldsymbol{u}, \boldsymbol{v}, \boldsymbol{w})$ produced from the GSVD of $\widetilde{\mathbf{K}}$ and $\mathbf{L}$ provide a ready DEM solution $\xi_{\lambda}$ as a function of the regularization parameter $\lambda$ (Hansen 1992), i.e.

$\xi_{\lambda}(T)=\sum_{i=1}^{6} \frac{\phi_{i}^{2}}{\phi_{i}^{2}+\lambda}\left(\frac{\left(\widetilde{g} \cdot \boldsymbol{u}_{i}\right) \boldsymbol{w}_{i}}{\gamma_{i}}\right)$,

where $\phi_{i}=\gamma_{i} / \beta_{i}$. To find the regularization parameter $\lambda$ that produces a solution $\xi_{\lambda}$ that matches the required $\chi^{2}=1$ (in data space) we use coarser sampling of $\lambda$ than in the original software, which helps with the computational speed increase. Even with this approach we achieve $\chi^{2}$ of typically between 0.5 and 1.75 with our regularized solutions, which are identical (within the error bars) to the results from the previous code. The above procedure is run twice (as in the original code) with the result from the first run $\xi_{\lambda}(T)$ being used to weight the constraint matrix as $\mathbf{L}_{j j}^{2}=d \log T_{j} / \xi_{\lambda}\left(T_{j}\right)$ before the second GSVD is performed and final $\xi_{\lambda}$ solution found. The horizontal errors are again taken to be the spread of the resolution matrix $\mathbf{R}_{\lambda} \mathbf{K}$ for each temperature bin (Doicu et al. 2010; Hannah \& Kontar 2012), where $\mathbf{R}_{\lambda}$ is the regularized inverse assumed to be close to the generalized inverse $\mathbf{K}^{\dagger}$, i.e. $\xi_{\lambda} \simeq \mathbf{R}_{\lambda} \boldsymbol{g}$. For the vertical error we do not use the Monte Carlo approach as in Hannah \& Kontar (2012) but take it to be $\Delta \xi(T)^{2}=\left\langle\delta \xi(T)^{2}\right\rangle=\left\|\mathbf{R}_{\lambda} \delta \boldsymbol{g}\right\|^{2}$. This produces a similar result but is considerably quicker to compute.

\section{DEM maps of the 2010-Nov-03 eruptive event}

\subsection{Data reduction}

The data between 12:12:02 UT and 12:19:02 UT (with a set of six different channel images every $12 \mathrm{~s}$ ) were prepared to level 1.6 by deconvolving the point spread function 
using aia_deconvolve_richardsonlucy.pro before being processed with aia_prep.pro. The co-alignment of the images were checked using aia_coalign_test.pro and found to be sub-pixel accurate. The resulting images were reduced down to sub-maps covering $-1150^{\prime \prime}$ to $-850^{\prime \prime}$ in the $x$ direction and and $-500^{\prime \prime}$ to $-200^{\prime \prime}$ in the $y$ direction, 501 by 501 pixels.

For each pixel position $(x, y)$ across the six different filters $(i=1 \ldots 6)$ in the 12 -s set provides the signal $g(x, y, t, i)$, in units of $\mathrm{DN} \mathrm{s}^{-1} \mathrm{px}^{-1}$, from which the associated errors $\delta g(x, y, t, i)$ are calculated using the readout noise and photon counting statistics. The set of six values and their associated errors, with the temperature response functions, are passed to the regularized DEM map code, as detailed in Sect. 2. The SDO/AIA temperature response functions are calculated using the CHIANTI fix, which is an empirical correction for missing emission in the CHIANTI v7.0 database (Dere et al. 1997; Landi et al. 2012a) in channels $94 \AA$ and $131 \AA$. The correction to match EVE spectroscopic full-disk observations, EVE normalization, is also used. For further details see ${ }^{2}$. CHIANTI v7.1 is now available, but the response functions using the new database have not been released yet. A preliminary investigation shows that this changes the response functions at lower temperatures $(<3 \mathrm{MK})$ but in a slightly different manner to the CHIANTI fix. We would not expect this to dramatically change the results presented in this paper, particularly those at higher temperatures. We use fairly broad temperature binning, twelve pseudo-logarithmic bins between 0.5 to $32 \mathrm{MK}$, so as to avoid a large number of temperature bins slowing down the code. These are larger than the designed achievable temperature resolution (Judge 2010), yet narrow enough to recover test models from testing the recovery of Gaussian model DEMs, as in Hannah \& Kontar (2012).

\subsection{DEM from individual pixels}

The resulting DEMs found for eight distinct pixel locations in the images from Fig. 1 are shown in Fig. 2. These pixels are shown for the $131 \AA$ and $211 \AA$ images, but the data from all six coronal-filter images were used to recover the DEMs. The vertical and horizontal errors shown are calculated as explained in Sect. 2.2. The vertical error is just the linear propagation of the uncertainty on the source data, but the horizontal error is more complicated since it gives a measure of the temperature spread (from the resolution matrix), as well as the quality of the regularized solution (i.e. strong off-diagonal terms) in each bin (for further details see Hannah \& Kontar 2012). The DEMs of the different positions show a variety of forms, but clearly none are isothermal, and the majority cannot be represented by a single Gaussian model. Shown for comparison are the DEMs obtained in these pixels before the eruption at 12:12:02 UT.

For each specific pixel we have

Pixel 1 - Core of the erupting plasmoid: two distinct components, one peaking in the range 1.5-3 MK, the other at 8-14 MK. The lower temperature one is likely the background corona along the line-of-sight, whereas the hotter is from the plasmoid itself. The pre-eruption DEM has a weaker high temperature component, and the highest temperature range is zero within the error bars;

Pixel 2 -Filament/stem behind the plasmoid: there are again two components with similar temperature ranges to the core of the plasmoid, but the low temperature component has more emission (due to higher densities lower in the corona

\footnotetext{
http://SSW/sdo/aia/response/chiantifix_notes.txt
}

compared to Pixel 1), and the higher temperature one has considerably weaker emission (due to a smaller amount of heated material accumulating in this location compared to the core, Pixel 1). The pre-eruption DEM has a weaker high temperature component, and the highest temperature range is zero within the error bars;

Pixel 3 - High corona away from the event: a single component over 1-3 MK with expected weak emission, owing to the lower density higher in the corona, and identical (within the errors bars) to the pre-eruption DEM;

Pixel 4 - Corona away from the event: a broader DEM with slightly higher peak emission than Pixel 3, likely due to it being lower in the corona but again peaking over 1-3 MK, and similar (within the errors bars) to the pre-eruption DEM;

Pixel 5 - Corona near the event: higher emission and broader DEM than the similar location of Pixel 4 indicating the presence of more hot coronal loops. The emission at high temperatures (above $6 \mathrm{MK}$ ) is zero within the errors bars. The preeruption DEM is slightly higher, though consistent within the errors, so it may indicate a slightly hotter and denser loop(s) moving out of this region;

Pixel 6 - Low corona flare emission: very high emission across all temperatures with a bright peak at 1.5-3 MK and 8-14 MK for the flare-heated material expanding up above the limb to be visible. The pre-eruption DEM shows emission across 6-8 MK and 8-11 MK, but it is weaker at higher temperatures, which might be due to the behind-the-limb flare;

Pixel 7-Envelope just ahead of the plasmoid: weaker emission than the core of the plasmoid (Pixel 1) but again a similar DEM with two components, peaking at 1.5-3 MK and $8-14 \mathrm{MK}$, the latter likely due to the accumulation of hotter coronal emission in this leading front of the eruption. The pre-eruption DEM does not show this hotter (8-14 MK) emission;

Pixel 8-Further ahead of the plasmoid: similar to the low temperature component of Pixel 7 (which is physically closer to the core of the erupting material) but no higher temperature component. It is consistent (within the error bars) to the pre-eruption DEM.

Overall the DEMs in Fig. 2 present a similar scenario to what was found by previous authors (Cheng et al. 2011; Bain et al. 2012) who were studying the behaviour just in the different SDO/AIA channels, as well as the DEM analysis from Cheng et al. (2012). Given the inherent difficulties in recovering DEMs, this is reassuring that their bulk features are consistent with the original SDO/AIA data. Here we have a hot (8-11, 11-14 MK) plasmoid core, well-defined with a filamentary stem-like structure behind it, similarly hot. This connects down to the flare loops filling up with even hotter material (14-19 MK), which have expanded just above the limb, consistent with the classic flux-rope eruption model. At cooler temperatures there is strong emission ahead of the erupting plasmoid, but there is also a lineof-sight coronal component (peaking about 1.5-2 MK) in all the pixels chosen.

\subsection{DEM maps}

To get a better understanding of the spatial structure of the event, we can create DEM maps for each temperature range, shown in Fig. 3. Many pixel locations in these maps show emission across a broad range of temperatures, highlighting the complex, multi-thermal nature of the line-of-sight DEMs. The temperature 

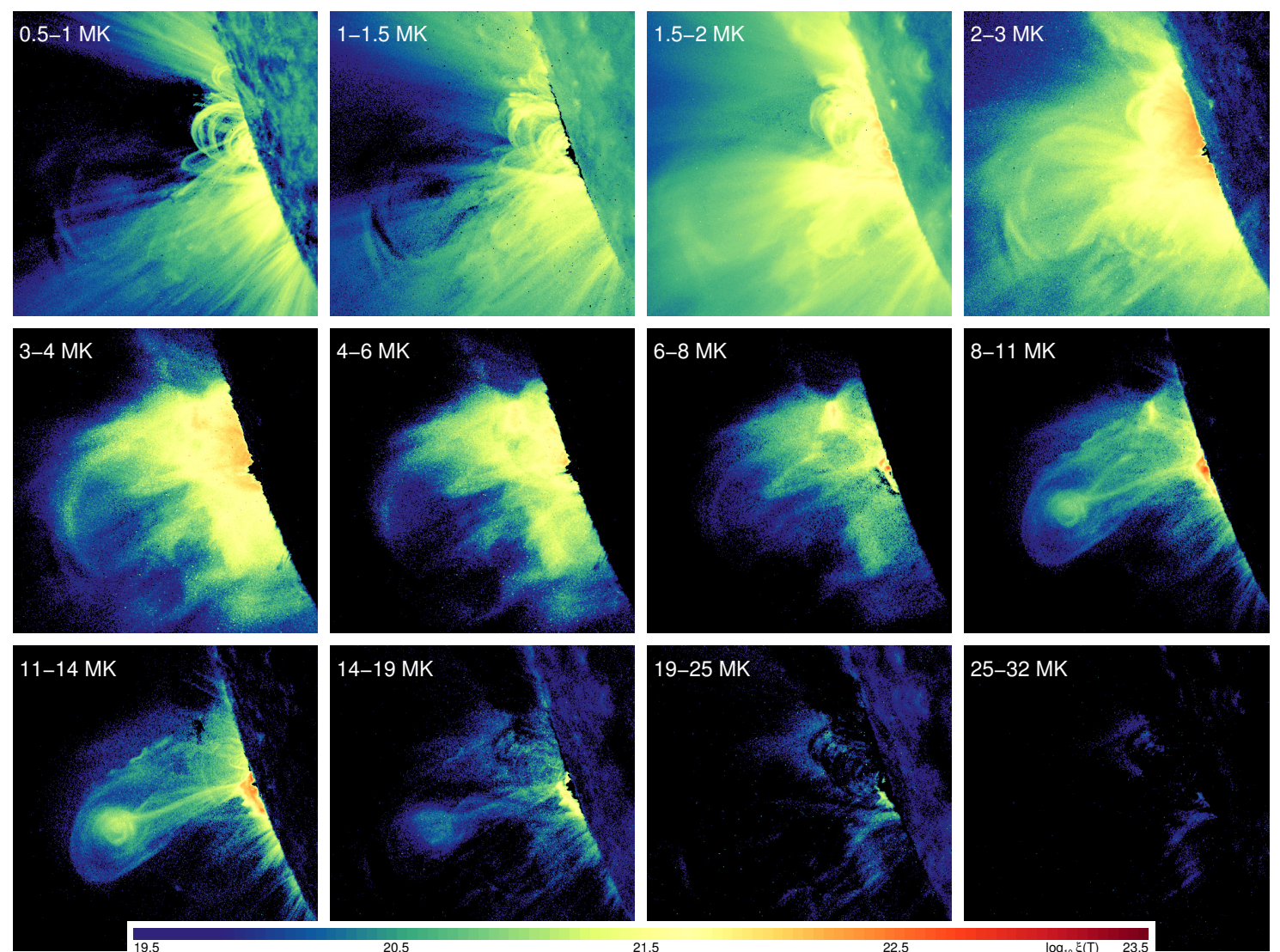

Fig. 3. DEM maps in different temperature ranges for the 3-Nov-2010 flare at 12:15:02 UT. The same pixel position in the twelve maps corresponds to the regularized DEM solution $\xi(T)$ (as shown in detail for eight pixel positions in Fig. 2) to the set of data values from the corresponding pixel in the six images shown in Fig. 1. The colour scale is $\log \xi(T)$ and is in units of $\mathrm{cm}^{-5} \mathrm{~K}^{-1}$.

maps for $0.5-1 \mathrm{MK}$ and $8-11 \mathrm{MK}$ do resemble the images from the $171 \AA$ and $131 \AA$ channels, respectively showing that the emission here is mostly from one component of the channels' temperature response. However, for the other temperature maps, features start to appear that are not apparent in the SDO/AIA images themselves, such as there being little 4-6 MK emission about the core of the erupting plasmoid. This emphasizes the complicated temperature response of SDO/AIA filters and the necessity of the DEM inference to interpret the temperature or density structures in the corona. Before discussing these spatial properties, it is prudent to investigate which of the regularized DEMs are robust solutions.

One major advantage of our regularization approach is that it produces information about the vertical $\Delta \xi(T)$ and horizontal $\Delta \log T$ uncertainty in the regularized solution (see Sect. 2.2), so we can plot uncertainty maps, shown in Fig. 4. The vertical error $\Delta \xi(T)$ (top row, given as a percentage error of the DEM) shows the largest uncertainties are predominantly from the regions where the emission measure is very low, and at the highest temperatures. The latter is due to a combination of SDO/AIA having lower sensitivity to the temperatures $>20 \mathrm{MK}$, and there also being little plasma emitting in this temperature range. the data, and also DEM, are therefore very noisy. Conversely, at the temperatures where SDO/AIA has a very strong response (about 0.5 to $3 \mathrm{MK}$ ) and there is bright solar emission, the signal, hence DEM, has a very small associated vertical error.

The horizontal error in the solution (bottom row Fig. 4) are mostly slightly larger than the SDO/AIA minimum theoretical limit of temperature resolution $\Delta \log T=0.1$ (Judge 2010). The largest uncertainties again come from regions where there is a weak response and/or noisy data, and this is particularly evident for the largest temperatures. But there are also positions where the data is noisy in the hottest temperature bands yet the horizontal error is small. This is due to the regularization approach having robustly determined that there is very weak or no emission at these positions and temperatures. Where the horizontal errors (temperature resolution) are small, it means that the emission is confined to that temperature range, but with large errors $(\geq 0.3)$ it suggests the emission shown in that temperature band could actually be attributed to neighbouring temperature bins. Therefore at temperatures where the horizontal uncertainty are large, the DEM solution should be treated with caution (see Hannah \& Kontar 2012), and additional analysis of the residuals could be useful (e.g. Piana et al. 2003).

Using this error information we can restrict the DEM maps to only show the values with the smallest vertical and horizontal errors, and this is shown for $\Delta \xi(T) \leq 30 \%$ and $\Delta \log T \leq 0.25$ in Fig. 5. At the hottest temperatures (19-25, 25-32 MK), there is virtually no emission owing to the largest uncertainties and poor confidence in the DEM solution, and any weak emission shown is likely unreliable. In 14-19 MK, the emission is predominantly coming from the flare heated material that has risen above the limb though there is a hint of very faint emission from the plasmoid core. These loops show the strongest emission in 8-11 and 11-14 MK, but also in these temperature ranges is the plasmoid core, an envelope of surrounding material and the filamentary stem behind it, all sharply defined. The plasmoid core 
I. G. Hannah and E. P. Kontar: Multi-thermal dynamics and energetics of a coronal mass ejection
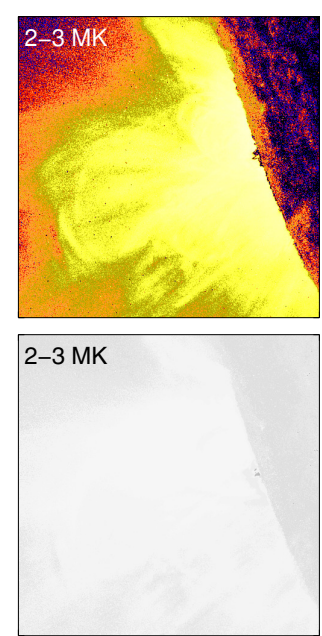

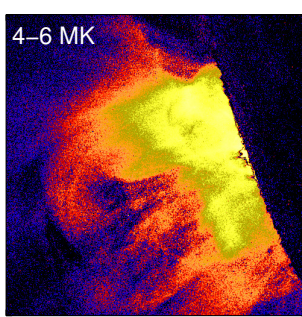

$4-6 \mathrm{MK}$

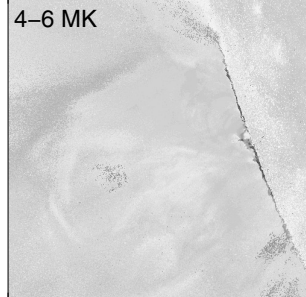

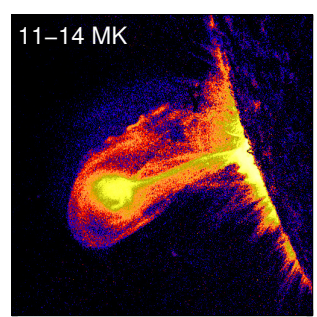

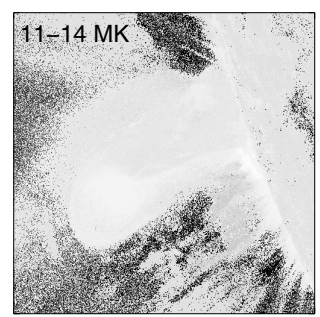

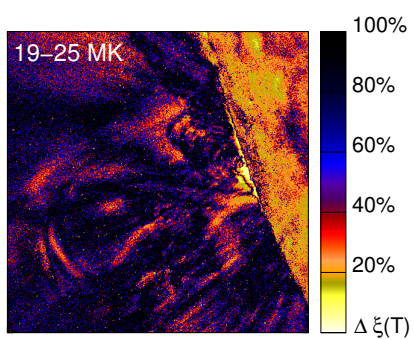

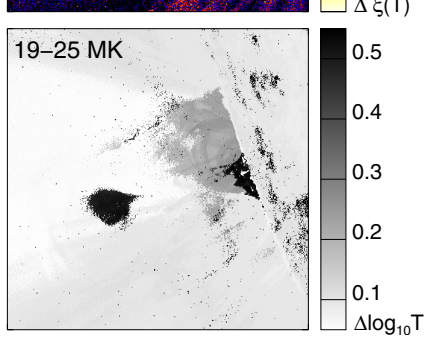

Fig. 4. Maps of the (top row) vertical uncertainty $\Delta \xi(T)$ and (bottom row) horizontal uncertainty, or temperature resolution $\Delta \log T$, in the regularized DEM solution shown in Fig. 3 for four temperature ranges.
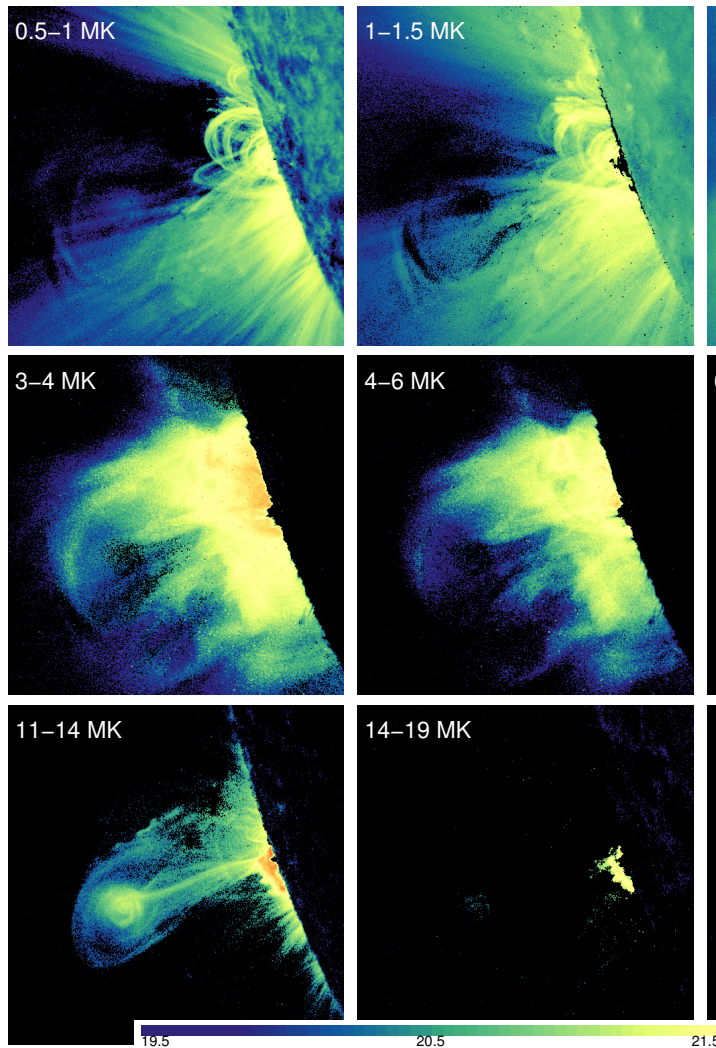
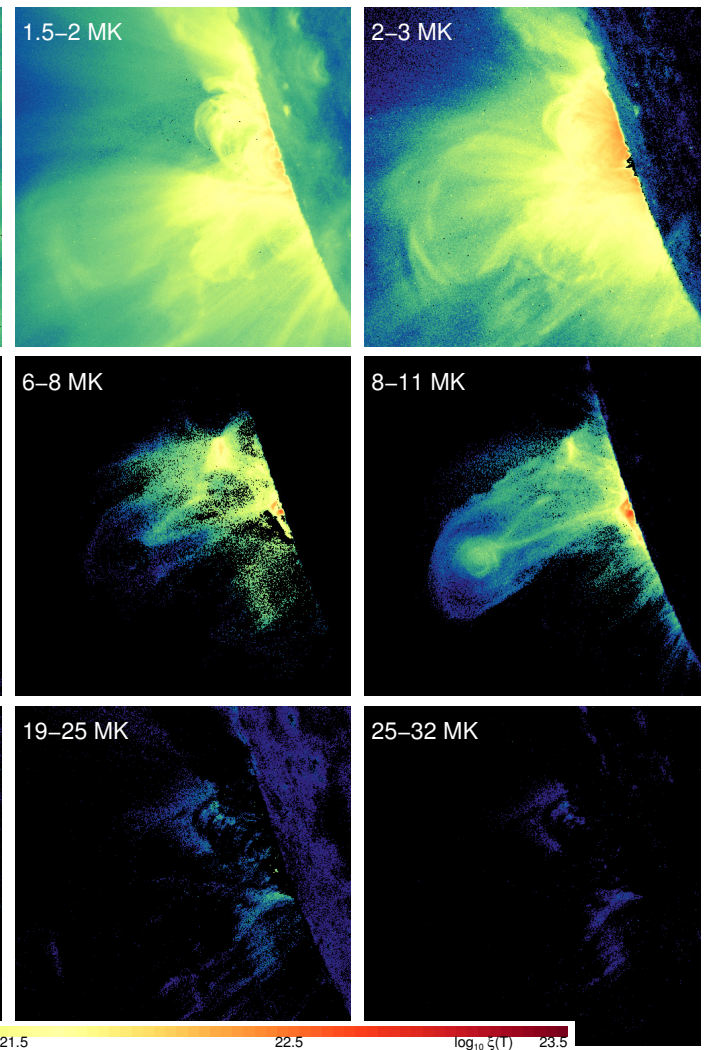

Fig. 5. DEM maps in different temperature ranges for the 3-Nov-2010 flare at 12:15:02 as shown in Fig. 3 but only showing pixels where $\Delta \xi(T) \leq$ $30 \%$ and $\Delta \log T \leq 0.25$. The colour scale is $\log \xi(T)$ and is in units of $\mathrm{cm}^{-5} \mathrm{~K}^{-1}$.

has slightly stronger emission in 11-14 MK than 8-11 MK. Only a single thin stem structure is clearly defined in 11-14 MK, but two are visible in 8-11 MK in a V-shape diverging away from the plasmoid core. In both cases their high temperatures, and the inflow region to these stems, is not explained by the classical flux rope eruption model. Some additional heating may be present, and the Kelvin-Helmholtz waves to the top of the erupting material are a signature of a reconnection layer surrounding the erupting flux rope, producing a secondary means of releasing energy (Foullon et al. 2011, and in prep.).
At cooler temperatures $(\leq 6 \mathrm{MK})$ the core of the plasmoid disappears, which is likely the cause of the dimming observed in some SDO/AIA images but it is not clear whether this is due to heating or mass loss. To try and understand this we look at the time evolution of the maps in Sect. 3.4. The V-shaped structure of the stems is still present though, clearly in 6-8 MK but slightly obscured by the bright surrounding coronal emission in 3-4 and 4-6 MK. Ahead of the plasmoid core we start to see the leading edge of a front of material, visible in 2-3, 3-4 and 4-6 MK. This density increase of material is likely due to a compression 

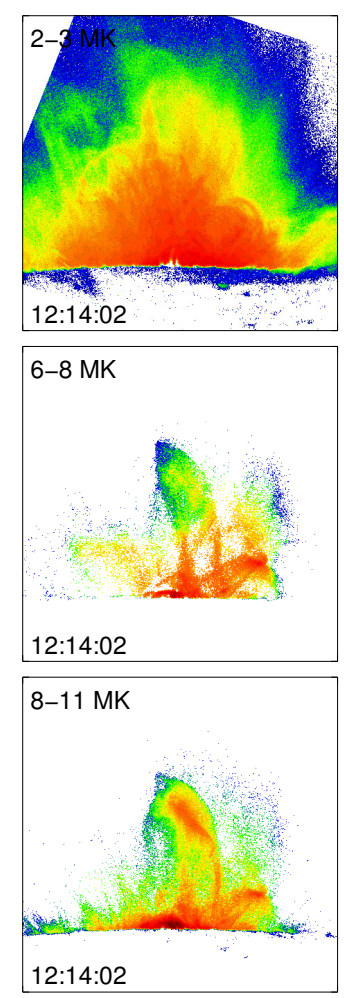
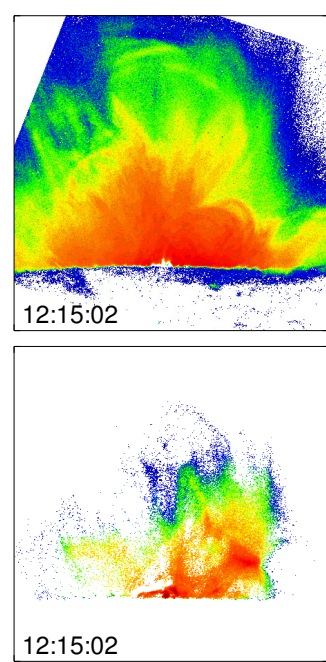

12:15:02

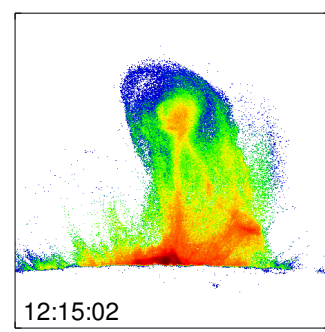

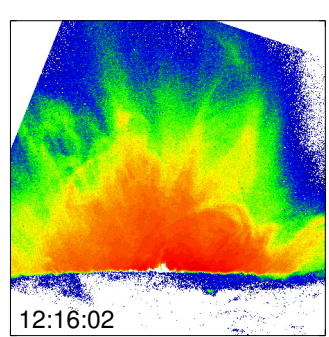
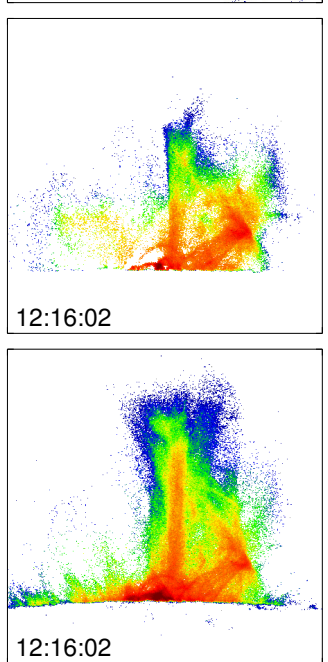
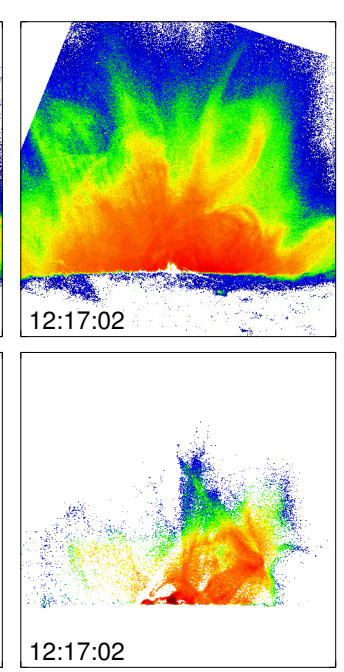

$12: 17: 02$

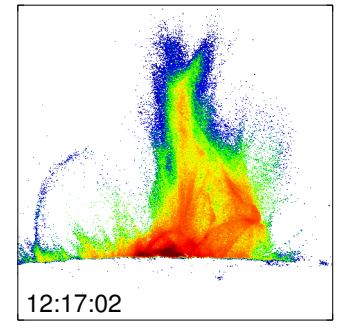

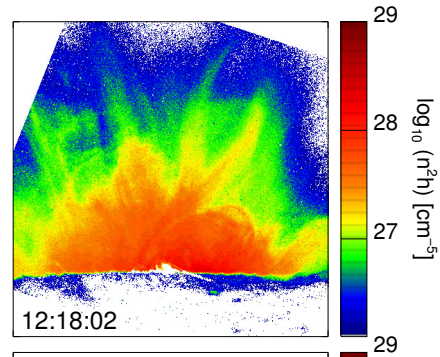
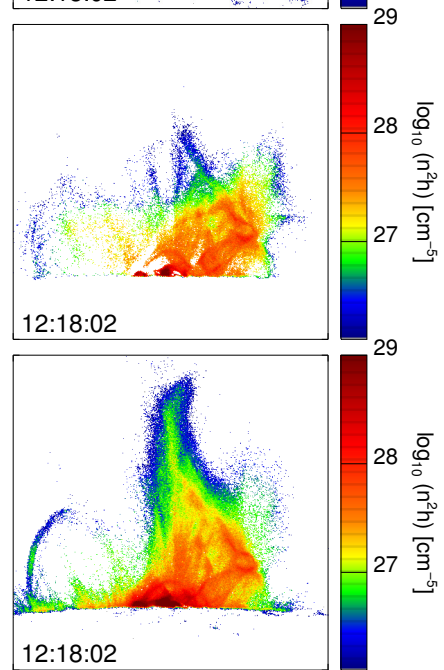

Fig. 6. Emission measure maps for various times in 2-3 MK, 6-8 MK, and 8-11 MK (top, middle, bottom rows). These maps have been rotated by $110^{\circ}$. The second column is of the time interval shown in previous figures. A movie of this is available with the electronic version of this paper, covering the 1-1.5 to 14-19 MK from 12:12:02 UT to 12:19:52 UT, see Fig. A.1.

front being driven ahead of the erupting plasmoid. At the coolest temperatures $(0.5-1,1-1.5$ and $1.5-2 \mathrm{MK})$ there is some faint evidence of the leading edge moving out but it is dominated by the emission from several hot coronal loops associated with the occulted active region and flare.

\subsection{Time evolution}

As the 2010-Nov-03 solar eruption is highly dynamic, we investigate the DEM maps as a function of time, which we have shown in three temperature ranges at one-minute intervals in Fig. 6. A movie of this is available with the electronic version of this paper, covering 1-1.5 to 14-19 MK from 12:12:02 UT to $12: 19: 52$ UT, see Fig. A.1. We have also converted the DEM maps into EM maps by removing the factor $\ln 10 T \mathrm{~d} \log T$, resulting in the values shown being $E M=n^{2} h\left[\mathrm{~cm}^{-5}\right]$, making it easier to calculate densities (see Sect. 3.5). Again we have only shown the emission in the pixels and temperature ranges that have $\Delta \xi(T) \leq 30 \%$ and $\Delta \log T \leq 0.25$, as in Fig. 5 .

In the 2-3 MK temperature range (top row Fig. 6) we see the leading edge of the eruption clearly at 12:15:02 UT, but afterwards gone, and the loops bending/collapsing back. There is some strong emission ahead of erupting flux rope at 12:14:02 UT but that disappears at later times, likely erupted. Lower in the corona the loops barely change over time, and only the highest ones are modified, being bent back after the eruption and leaving a void where the stem forms at higher temperatures. In 6-8 MK (middle row Fig. 6) we see the initial eruption of the plasmoid core and stems behind it at 12:14:02 UT, but at later times there is a void of material at this temperature, again suggestive of material loss (or spreading out) due to the eruption mass loss. There is increased emission from the loops that have bundled up below the eruption to the right indicating possible heating of material in these contracting loops.

The erupting plasmoid and the filamentary stem structure connecting it to the lower solar atmosphere is sharply defined in $8-11 \mathrm{MK}$ (bottom row Fig. 6). The strong emission from the core of the plasmoid quickly weakens as it moves higher, leaving the strongest emission from the vertical stem behind it. It would be tempting to immediately classify this structure as a current sheet, given the superficial similarity to the classical eruption model. However, this stem is relatively thick and appears to be made up of several thinner structures. This may be a projection effect with multiple thin stem misaligned along the line-of-sight. A bigger issue is that this structure shows strong emission in this hot (11-14 MK) temperature range, and again the classical model is unable to explain the presence of such hot material flowing in to the current sheet. Below to the right of the eruption (or to the top right in the original orientation), hot loops bundle up between 12:16:02 UT to 12:18:02 UT. This is associated with Kelvin-Helmholtz waves (Foullon et al. 2011) and possible density increases due to outflowing material (Savage et al. 2012). In the classic eruptive model this region would be associated with shrinking loops and the outflow from the reconnection region. However, the dynamics here are clearly more complicated and difficult to unambiguously ascertain due to the strong emission lower in the atmosphere. In the 12:17:02 UT and 12:18:02 UT images another large coronal loop develops to the left of the main eruption. This fainter emission in 11-14 MK is likely flare-heated material that has expanded into this large loop. STEREO-B/SECCHI images from behind the limb do show a separate flaring region located at the base of this hot loop (Savage et al. 2012). 


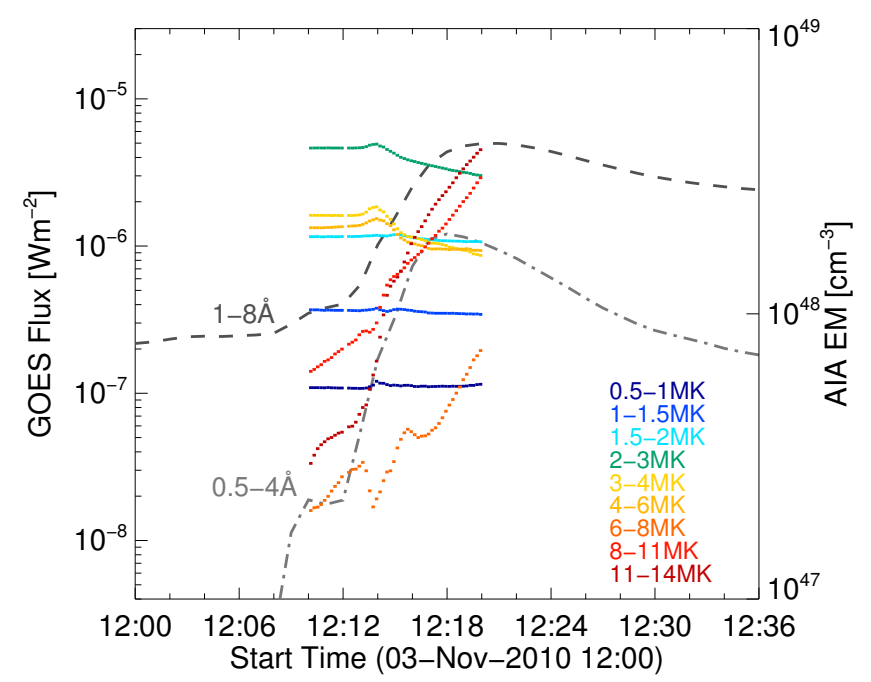

Fig. 7. GOES SXR lightcurve of the 3-Nov-2010 flare C4.9 (grey lines) with the spatially integrated emission measures derived from the SDO/AIA observations for the chosen temperature ranges (coloured lines).

By summing over the whole of the region shown in Fig. 6, we can produce a time profile in each temperature band, shown in Fig. 7 in comparison to the GOES SXR emission. We have computed this between 12:12:02 UT and 12:19:50 UT (the time range shown in the movie available in the electronic version of this paper, see Fig. A.1) as saturation from the flaring loops starts to dominate and produces unreliable EM maps in these regions. From Fig. 7 we see that there is a slight increase in most of the temperature ranges as the eruption begins (12:13 UT), which then drops off as the material is ejected (12:15 UT), suggesting this initial change is due to the heating, then the loss of coronal plasma. After this the hottest temperature ranges (6-8, 8-11, and 11-14 MK) increase in a similar manner (though slightly delayed) to the SXR emission observed by GOES. This is initially due to further heating in the current sheet and the shrinkage of loops below the eruption with heated material flowing out of the reconnection region. The later sharp increase is likely due to the bright post-flare loops rising above the limb. At the cooler temperatures it will be later in the event before the current sheet and flare-heated material (if visible above the limb) cool into these temperature ranges. This explains the decrease in the emission at these temperatures after the loss of material during the eruption. The very small decrease at the lowest temperatures $(0.5-1$, $1-1.5,1.5-2 \mathrm{MK}$ ) suggest that this emission is dominated by the background and line-of-sight coronal emission over the whole region.

To investigate the time profile of the erupting material in different temperature ranges, we have manually tracked the front of the emission in running difference maps of the emission measure, i.e. $\xi\left(T, x, y, t_{i}\right)-\xi\left(T, x, y, t_{i-1}\right)$. This is shown in Fig. 8 for 2-3 MK and 11-14 MK, with black indicating a decrease in emission, white an increase, and the colour lines showing the manually tracked front of the eruption. This type of analysis has been performed before but in individual SDO/AIA wavelength channels (Cheng et al. 2011; Bain et al. 2012) and not for specific temperature ranges. The former has the advantage of a clearer time evolution as the channels are $\mathrm{a} \approx 3 \mathrm{~s}$ duration image every $12 \mathrm{~s}$, whereas the EM maps are effectively $12 \mathrm{~s}$ cadence due to the use of six different channels in their calculation. This makes the determination of the leading edge tricky.
The resulting height (above an arbitrary position) as a function of time for the different temperature ranges are shown in Fig. 9. Here we have linearly fitted the data to produce a velocity as a function of temperature. These velocities and associated error (the largest between the error in the fitted parameter and from three SDO/AIA pixels travelled in 12 s) are shown in Fig. 10. In the cooler temperature range, the leading edge of the eruption travels at $1246 \mathrm{~km} \mathrm{~s}^{-1}$ in $0.5-1 \mathrm{MK}$ decreasing to $1029 \mathrm{~km} \mathrm{~s}^{-1}$ in 2-3 MK. This general trend of a slower eruption at higher temperature is more evident above 3-4 MK once we are in the range associated with the plasmoid eruption itself. From 3-4 MK to 11-14 MK, the velocities are consistently between $597 \mathrm{~km} \mathrm{~s}^{-1}$ to $664 \mathrm{~km} \mathrm{~s}^{-1}$. These two distinct structures at different velocities are consistent with those found by tracking the eruption in the SDO/AIA channels (Cheng et al. 2011; Bain et al. 2012), with the $131 \AA$ and $335 \AA$ at 667 and $747 \mathrm{~km} \mathrm{~s}^{-1}$ and the cooler $211 \AA$ and $193 \AA$ at 1154 and $1439 \mathrm{~km} \mathrm{~s}^{-1}$ (Bain et al. 2012).

\subsection{Density and thermal energy}

To recover the density from the EM maps, an estimate of the line-of-sight depth $h$ of the emission is required. As the structures are sharply defined at higher temperatures (visible in Fig. 11, labelling the three distinct components: plasmoid core, envelope, and stem), we can use their apparent width in the EM maps as the depth, assuming cylindrical geometry. We focus only on 11-14 MK here because the structures are similar in shape to those in 8-11 MK (bottom row of Fig. 6), and at lower temperatures this would be difficult since the shape of the structures is far harder to estimate reliably. The density of the sheath of the Kelvin-Helmholtz waves has been estimated at lower temperatures (about $4 \mathrm{MK}$ ) using emission measures found with both our regularized approach and Gaussian model forward-fitting techniques, finding similar values, and is the subject of a separate publication (Foullon et al., in prep.).

The EM maps in 11-14 MK for five time intervals during the plasmoid eruption are shown in Fig. 12 (top row). Here we have plotted the EM as a function of position (bottom row) for various slices through the erupting structure, indicated in the EM maps by dotted horizontal lines. From these slices we can determine a width of the structures of interest: the erupting plasmoid core, envelope, and stem. Assuming a cylindrical geometry in these structure we can use the width as the depth, giving the line-ofsight extent $h$. Since $E M=n^{2} h$, this readily gives an estimate of the density in the structure and these values for the various structures at the different times are given in Table 1 . Here we see that the density in the core region (about $3 \times 10^{9} \mathrm{~cm}^{-3}$ ) does not change between the two time intervals shown before it fully erupts. The envelope of material surrounding the plasmoid core has a lower density $\left(9 \times 10^{8} \mathrm{~cm}^{-3}\right)$ but still shows a sharp transition to the background corona because that has effectively no emission in this temperature range.

The density in the stem region does decrease over time, but that is initially due to a widening of the structure (seen at 12:16:02 UT onwards) rather than a reduction in the emission measure. As discussed before, it is difficult to attribute such a wide hot structure to the monolithic current sheet as proposed by the classic eruption model. If we instead assume the structures are more elongated, using a line-of-sight component of $h=100 \mathrm{Mm}$ (shown in Table 1), we find that the density of the stem does not vary much with time. In fact these slightly lower densities (about $10^{9} \mathrm{~cm}^{-3}$ ) are similar for both the core and stem regions. 

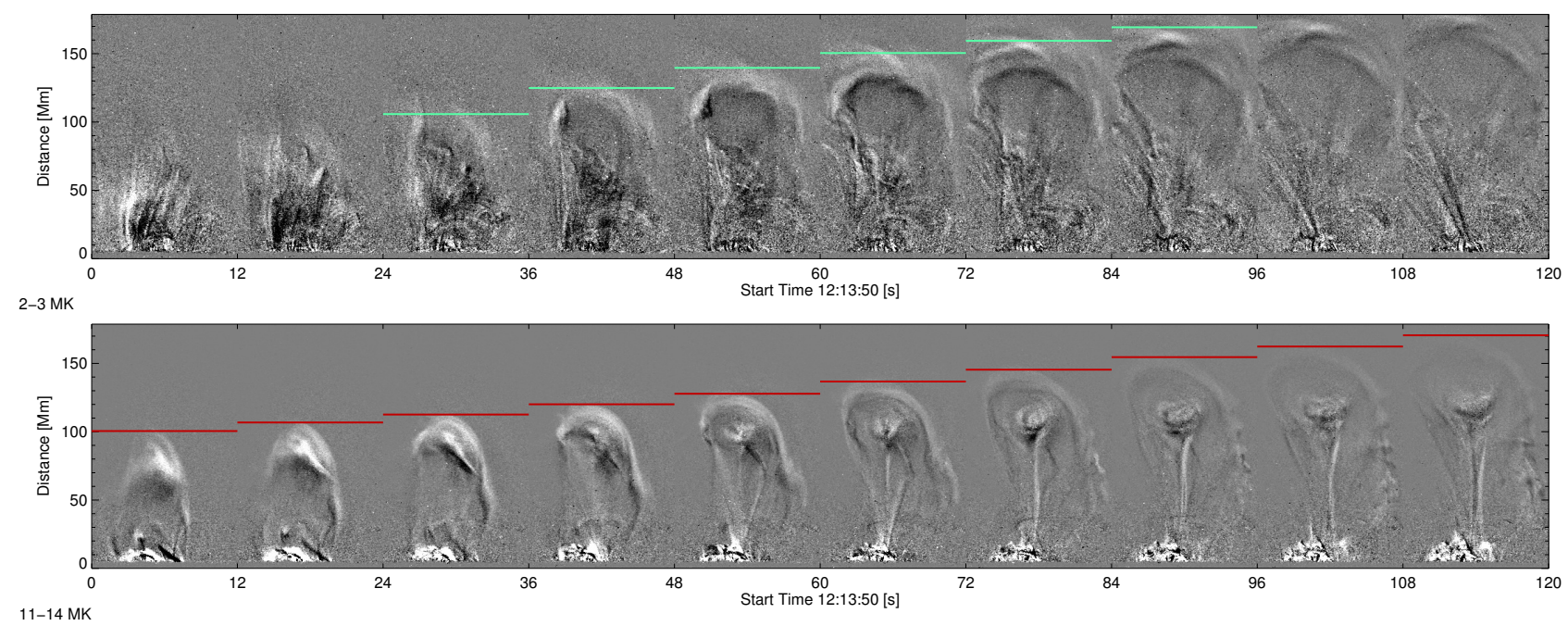

Fig. 8. Running differences of the DEM maps $\left(\xi\left(T, x, y, t_{i}\right)-\xi\left(T, x, y, t_{i-1}\right)\right)$ for 2-3 MK (top row) and 11-14 MK (bottom row), with white showing an increase in DEM, black a decrease. The leading edge of the erupting plasma was tracked and is indicated by the red horizontal lines The resulting height profiles as a function of time for these and other temperature ranges is shown in Figs. 9 and 10. These maps have been rotated by $110^{\circ}$.

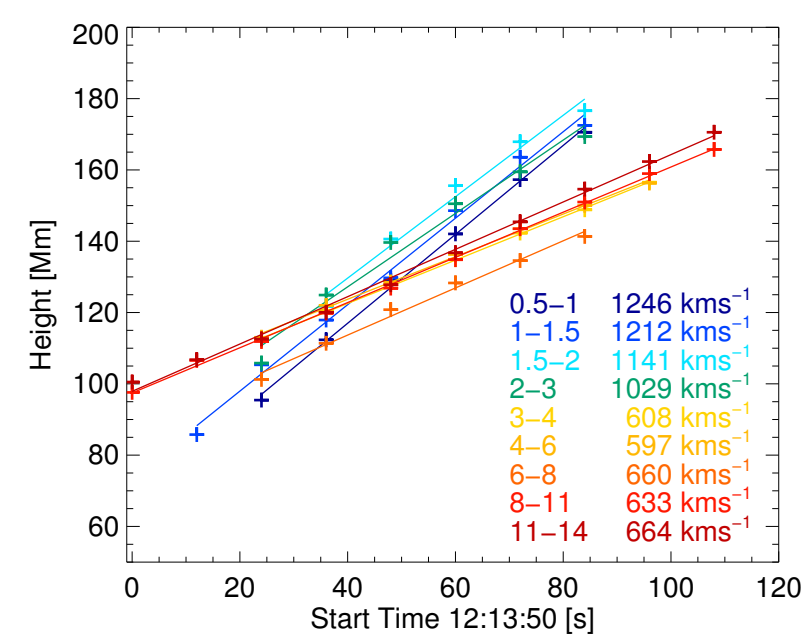

Fig. 9. Height (above an arbitrary origin) versus time profiles of the leading edge of the erupting plasma for each of the temperature ranges where the propagation could be reliably tracked.

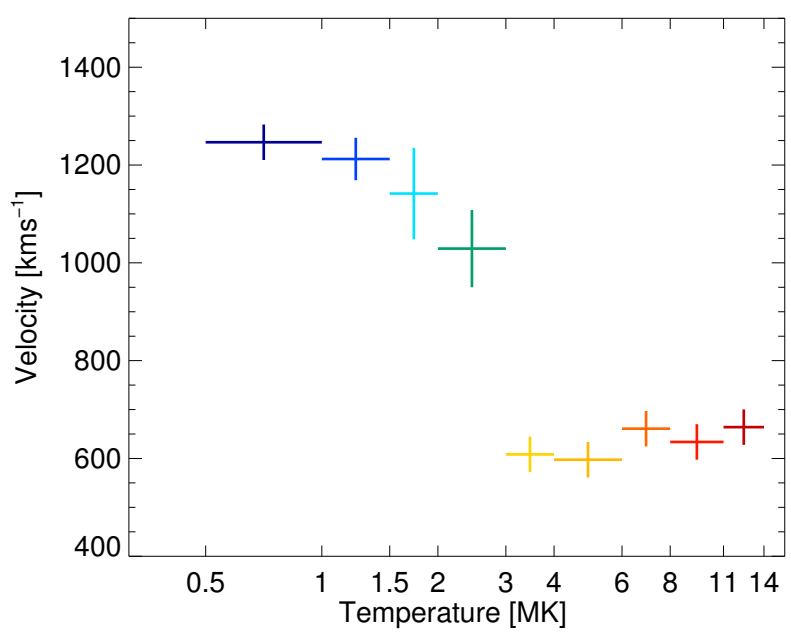

Fig. 10. Velocity of the leading edge of the eruption as a function of temperature, obtained from linear fits to the data shown in Fig. 9. The vertical uncertainty is taken as the largest of the error in the parameter from the linear fits shown in Fig. 9 and three AIA pixels travelled in $12 \mathrm{~s}$.

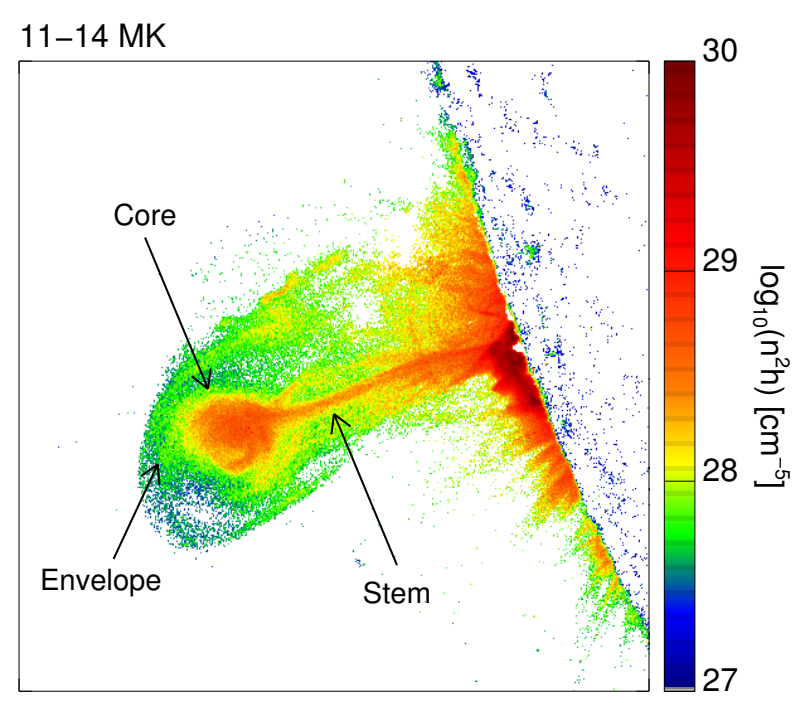

Fig. 11. Emission measure map for 11-14 MK at 12:15:02 UT with the different components identified.

The thermal energy of this emission can be calculated via $W_{\mathrm{T}}=3 n V k_{\mathrm{B}} T$, where we take $T=12.5 \mathrm{MK}$ as the middle of the temperature range. We estimate the volume using $V=w h L$, with $w$ the width, $h$ the line-of-sight component, and $L$ the extent perpendicular in the image to $w$. This is found using a filling factor of unity, and because this could be smaller, the values we calculate are upper limits to the thermal energy. The energies are shown in Table 1 for the times and regions in Fig. 12. We find that the thermal energy in this temperature range as the core erupts increases slightly $\left(4.1\right.$ to $\left.5.1 \times 10^{29} \mathrm{erg}\right)$, whereas the energy in the stem structure greatly increases just after the impulsive eruption ( 1.2 to $9.1 \times 10^{29} \mathrm{erg}$ ). This is due to the widening of the stem whilst maintaining the same emission measure per pixel within the stem. This weakens by 12:18:02 UT when the emission measure starts to drop and the stem narrows. If we again assume the structures are elongated, with line-of-sight $h=100 \mathrm{Mm}$, we get a smaller variation between the thermal energy of the different structures and how they evolve with time, with energies of about $10^{30} \mathrm{erg}$. In both cases there is an increase 
Table 1. Thermal parameters for the different features shown in Figs. 11 and 12.

\begin{tabular}{|c|c|c|c|c|c|c|c|c|c|c|c|}
\hline \multirow[b]{2}{*}{ Time } & \multirow[b]{2}{*}{ Feature } & \multirow[b]{2}{*}{$\begin{array}{c}E M \\
10^{28} \mathrm{~cm}^{-5}\end{array}$} & \multirow[b]{2}{*}{$\begin{array}{c}\text { Width } \\
\text { Mm }\end{array}$} & \multicolumn{3}{|c|}{$(h=w)$} & \multicolumn{2}{|c|}{ Energy } & \multirow{2}{*}{$\begin{array}{c}(h=100 \mathrm{Mm}) \\
\text { Density } \\
10^{9} \mathrm{~cm}^{-3}\end{array}$} & \multicolumn{2}{|c|}{ Energy } \\
\hline & & & & $\begin{array}{c}\text { Length } \\
\mathrm{Mm}\end{array}$ & $\begin{array}{l}\text { Density } \\
10^{9} \mathrm{~cm}^{-3}\end{array}$ & $\begin{array}{c}\text { Vol } \\
10^{28} \mathrm{~cm}^{3}\end{array}$ & $\begin{array}{l}\text { Thermal } \\
10^{29} \text { erg }\end{array}$ & $\begin{array}{l}\text { Kinetic } \\
10^{29} \text { erg }\end{array}$ & & $\begin{array}{l}\text { Thermal } \\
10^{29} \text { erg }\end{array}$ & $\begin{array}{l}\text { Kinetic } \\
10^{29} \mathrm{erg}\end{array}$ \\
\hline $12: 14: 02$ & Core & 2.5 & 25 & 40 & 3.2 & 2.5 & 4.1 & 2.9 & 1.6 & 8.2 & 5.8 \\
\hline $12: 15: 02$ & Core & 3.9 & 30 & 30 & 3.6 & 2.7 & 5.1 & 3.6 & 2.0 & 9.3 & 6.5 \\
\hline & Envelope & 0.5 & 65 & 100 & 0.9 & 39.6 & 18.0 & 12.6 & 0.7 & 20.5 & 14.4 \\
\hline & Stem & 2.5 & 7 & 80 & 6.0 & 0.4 & 1.2 & & 1.6 & 4.6 & \\
\hline $12: 16: 02$ & Stem & 2.0 & 25 & 100 & 2.8 & 6.3 & 9.1 & & 1.4 & 18.3 & \\
\hline $12: 17: 02$ & Stem & 3.2 & 20 & 110 & 4.0 & 4.4 & 9.1 & & 1.8 & 20.3 & \\
\hline 12:18:02 & Stem & 1.6 & 10 & 130 & 4.0 & 1.3 & 2.7 & & 1.3 & 8.5 & \\
\hline
\end{tabular}
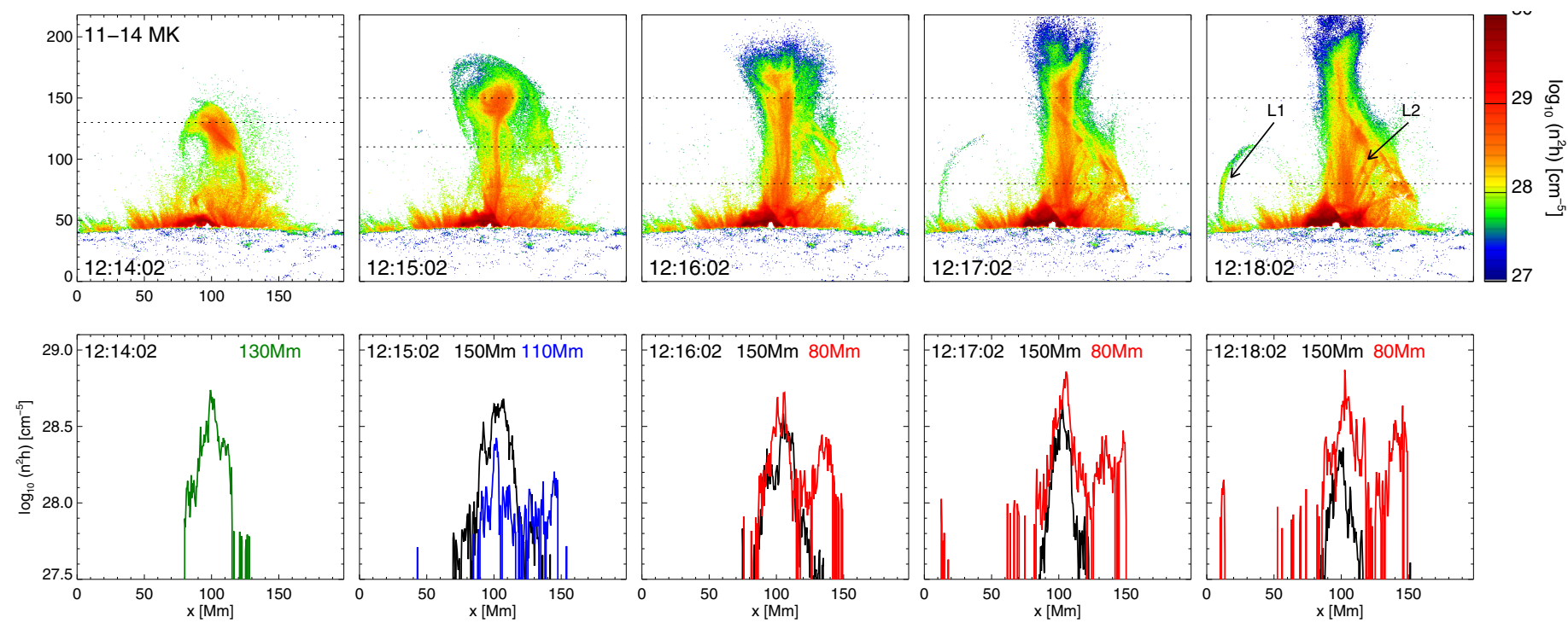

Fig. 12. Top: emission measure maps for various times in 11-14 MK. Bottom: the emission measure profile at various heights for one minute time intervals in 11-14 MK, indicated by the dotted lines in the top panel.

in the thermal energy of the stem that is consistent with the idea of continuous energy release in this region.

The envelope of hot material around the plasmoid core has considerably higher thermal energy which may be due to assumptions used when calculating the envelope volume. Here we have calculated the volume of the region minus the volume of the plasmoid core. If we instead consider the envelope as a thinner shell of $5 \mathrm{Mm}$, then we get a volume of $10^{29} \mathrm{~cm}^{3}$ and $1.6 \times 10^{29} \mathrm{~cm}^{3}$ for $h=w$ and $h=100 \mathrm{Mm}$. This would result in thermal energies about three to four times lower than those given in Table 1 and similar to those for the core. There is, however, significant uncertainty as to the thickness of the shell, and therefore the values in Table 1 are an upper limit for the parameters used.

We can also calculate the kinetic energy for the structures that propagate outwards, the plasmoid envelope and core. The kinetic energy is taken as $W_{\mathrm{K}}=n V m_{\mathrm{p}} v^{2} / 2$ where $m_{\mathrm{p}}$ is the proton mass, and the velocity is $v=660 \mathrm{~km} \mathrm{~s}^{-1}$ from Fig. 9 . These are again given in Table 1 for $h=w$ and $h=100 \mathrm{Mm}$. Here we find that the thermal energy (in 11-14 MK) is consistently slightly higher than the kinetic energy of the material in this temperature range. A higher thermal-to-kinetic energy has also been found previously by Landi et al. (2010). This similarity between the thermal and kinetic energies is typical of CMEs from observations higher in the corona (e.g. Forbes 2000). Our values for the energies are also lower than those for a "moderately" large CME (Forbes 2000) $\left(10^{30}\right.$ versus $10^{32} \mathrm{erg}$ ) which is expected as our energy is just from one component of the CME and in one temperature range.

\subsubsection{Coronal loop density}

In the later time intervals in Fig. 12 (12:18:02 UT), there are distinctive coronal loops associated with the flaring region (L1) and the shrinkage below the eruption (L2). Again assuming $w=h$ we can calculate the loop density, taking them to be about $2 \mathrm{Mm}$, though we do not estimate the energy because we cannot determine a reliable loop length. The large coronal loop (L1), associated with the core of the occulted flaring region, is visible from 12:17:02 UT onwards, and its density increases as more hot material fills the loop (from $5.6 \times 10^{9} \mathrm{~cm}^{-3}$ to $7.1 \times 10^{9} \mathrm{~cm}^{-3}$ ). The loops below the ejected material (L2) have an emission measure that doubles between 12:17:02 UT and 12:18:02 UT, resulting in a density increase to $1.3 \times 10^{10} \mathrm{~cm}^{-3}$ from $8.9 \times 10^{9} \mathrm{~cm}^{-3}$. This 
increase in heated material may be due to outflows from the reconnection region in the stem structure (Savage et al. 2012) or the reconnection layer above the loops where Kelvin-Helmholtz waves were located (Foullon et al., in prep.).

\section{Discussion and conclusions}

Regularized inversion of multi-filter observations provides an essential tool for studying the temporal and spatial evolution of multi-thermal plasma during SEEs. The regularized maps of an erupting plasmoid presented in this paper allow the basic plasma properties (integrated along the line-of-sight) to be inferred and the density and energetics of various parts of the eruption to be calculated. We find that the leading edge of the eruption travels between 1029-1246 $\mathrm{km} \mathrm{s}^{-1}$ and has the temperature range $\leq 2-3 \mathrm{MK}$ followed by slower $597-664 \mathrm{~km} \mathrm{~s}^{-1}$ but hotter plasma $\geq 3-4 \mathrm{MK}$. The erupting core and stem (which could be interpreted as a plasmoid with a trailing current sheet) appear sharply defined in 11-14 MK. The width of the stem/current sheet we find $(7-25 \mathrm{Mm})$ is far larger than expected in the classical reconnection model, with <1 Mm predicted (Vršnak et al. 2009). This value is, in fact, lower than those found higher in the corona (e.g. Ciaravella et al. 2002; Landi et al. 2012b), suggesting continuous expansions as the CME lifts up. The stem/current sheet width we determine is, however, at a higher temperature range and made with a higher spatial resolution observations than these previous results.

During the impulsive phase, we find that the density is about $3 \times 10^{9} \mathrm{~cm}^{-3}, 6 \times 10^{9} \mathrm{~cm}^{-3}, 9 \times 10^{8} \mathrm{~cm}^{-3}$ in the plasmoid core, stem, and surrounding envelope of material. This gives thermal energy estimates of $5 \times 10^{29} \mathrm{erg}, 1 \times 10^{29} \mathrm{erg}$ and $2 \times 10^{30} \mathrm{erg}$. These are slightly higher than the values found for the kinetic energy of the erupting envelope and core, which is consistent with previous CME energy estimations (Landi et al. 2010). The observations also show the increase in the thermal energy of the core (plasmoid) and of the stem (current sheet) as the CME is rising from 12:14:02 UT to 12:17:02 UT, suggesting ongoing energy release. The increase in energy is more noticeable for the stem with it more than doubling over three minutes. This is consistent with the predictions of recent reconnection models (e.g. Murphy et al. 2010; Reeves et al. 2010) with the energy released in the current sheet pumped into the plasmoid.

The reconstructed DEM maps show the multi-thermal nature of the corona, which is unsurprising given that we have a hot plasmoid erupting through the cooler surrounding corona, which are two distinct structures with different temperature characteristics. Our DEM maps show in greater detail the same basic physical picture that was found by Cheng et al. (2012) using a different DEM reconstruction method for a few regions in the CME; that is the bright leading front of the CME is composed of a coronalike temperature distribution, with the increased emission due to the accumulation of material, whereas the erupting core contains heated material (>8 MK). All DEM solutions should, however, be treated carefully given the uncertainties in the temperature response functions (O'Dwyer et al. 2010) and the possibly broad temperature resolution (Testa et al. 2012). Used carefully, with these issues kept in mind, regularized DEM maps present a useful tool for exploring the dynamics of heating in the solar corona with SDO/AIA observations.

Acknowledgements. This work is supported by STFC grant ST/I001808/1 (I.G.H., E.P.K.). Financial support by the European Commission through the FP7 HESPE network (FP7-2010-SPACE-263086) is gratefully acknowledged (E.P.K.). We would also like to thank the referee for the constructive comments.

\section{Appendix A: EM movie of the eruption}

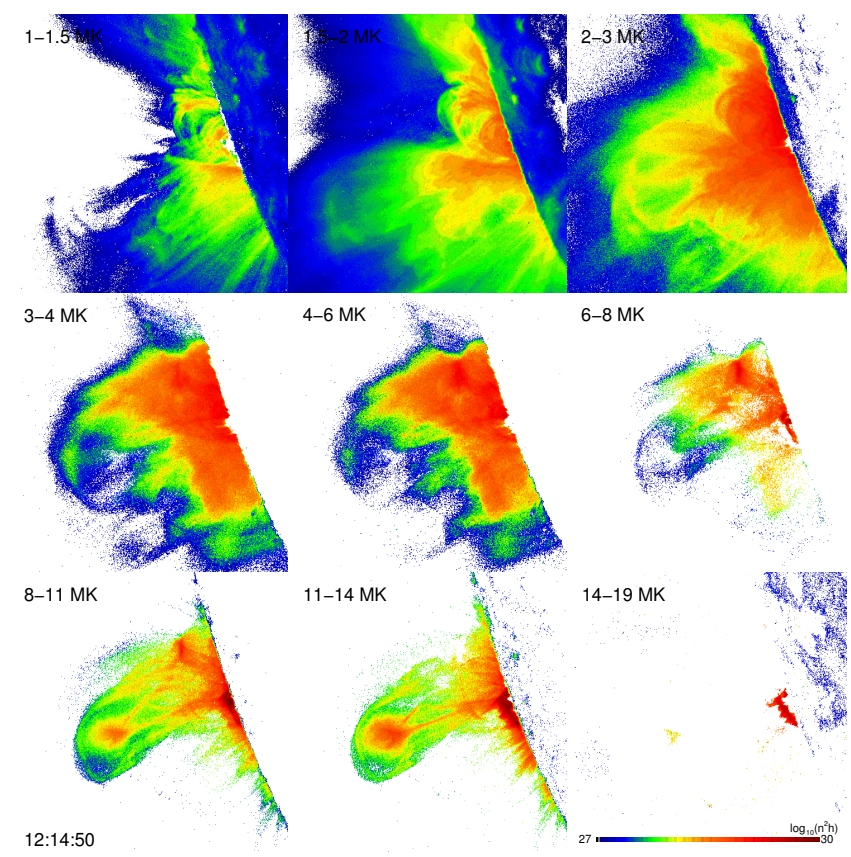

Fig. A.1. One frame of the movie, available online, showing the EM maps, $\log n^{2} h$ in units of $\mathrm{cm}^{-5}$, for the temperature ranges $1-1.5$ through to $14-19 \mathrm{MK}$, starting at 12:12:02 UT and running until 12:19:52 UT.

\section{References}

Archontis, V., \& Török, T. 2008, A\&A, 492, L35

Aschwanden, M. J., \& Boerner, P. 2011, ApJ, 732, 81

Aulanier, G., Török, T., Démoulin, P., \& DeLuca, E. E. 2010, ApJ, 708, 314

Bain, H. M., Krucker, S., Glesener, L., \& Lin, R. P. 2012, ApJ, 750, 44 Bemporad, A., Poletto, G., Suess, S. T., et al. 2006, ApJ, 638, 1110 Bertero, M., DeMol, C., \& Pike, E. R. 1985, Inverse Problems, 1, 301

Boerner, P., Edwards, C., Lemen, J., et al. 2012, Sol. Phys., 275, 41

Canfield, R. C., Hudson, H. S., \& McKenzie, D. E. 1999, Geophys. Res. Lett., 26,627

Carmichael, H. 1964, NASA SP, 50, 451

Cheng, X., Zhang, J., Liu, Y., \& Ding, M. D. 2011, ApJ, 732, L25

Cheng, X., Zhang, J., Saar, S. H., \& Ding, M. D. 2012, ApJ, 761, 62

Ciaravella, A., Raymond, J. C., Li, J., et al. 2002, ApJ, 575, 1116

Craig, I. J. D. 1977, A\&A, 61, 575

Craig, I. J. D., \& Brown, J. C. 1986, Inverse problems in astronomy (Bristol: Hilger)

Démoulin, P., \& Aulanier, G. 2010, ApJ, 718, 1388

Dere, K. P., Landi, E., Mason, H. E., Monsignori Fossi, B. C., \& Young, P. R. 1997, A\&AS, 125, 149

Doicu, A., Trautmann, T., \& Schreier, F. 2010, Numerical Regularization for Atmospheric Inverse Problems, Springer Praxis Books in Environmental Sciences (Springer)

Fan, Y., \& Gibson, S. E. 2003, ApJ, 589, L105

Fludra, A., \& Sylwester, J. 1986, Sol. Phys., 105, 323

Forbes, T. G. 2000, J. Geophys. Res., 105, 23153

Foullon, C., Verwichte, E., Nakariakov, V. M., Nykyri, K., \& Farrugia, C. J. 2011, ApJ, 729, L8

Frazin, R. A., Kamalabadi, F., \& Weber, M. A. 2005, ApJ, 628, 1070

Frazin, R. A., Vásquez, A. M., \& Kamalabadi, F. 2009, ApJ, 701, 547

Gibson, S. E., Foster, D., Burkepile, J., de Toma, G., \& Stanger, A. 2006, ApJ, 641,590

Gopalswamy, N., \& Hanaoka, Y. 1998, ApJ, 498, L179

Green, L. M., Kliem, B., \& Wallace, A. J. 2011, A\&A, 526, A2

Hannah, I. G., \& Kontar, E. P. 2012, A\&A, 539, A146

Hansen, P. C. 1992, Inverse Problems, 8, 849

Harrison, R. A., Bryans, P., Simnett, G. M., \& Lyons, M. 2003, A\&A, 400, 1071

Hirayama, T. 1974, Sol. Phys., 34, 323

Hudson, H. S., Bougeret, J.-L., \& Burkepile, J. 2006, Space Sci. Rev., 123, 13 
I. G. Hannah and E. P. Kontar: Multi-thermal dynamics and energetics of a coronal mass ejection

Innes, D. E., McKenzie, D. E., \& Wang, T. 2003, Sol. Phys., 217, 267

Judge, P. G. 2010, ApJ, 708, 1238

Kahler, S. W., Moore, R. L., Kane, S. R., \& Zirin, H. 1988, ApJ, 328, 824

Kashyap, V., \& Drake, J. J. 1998, ApJ, 503, 450

Ko, Y.-K., Raymond, J. C., Lin, J., et al. 2003, ApJ, 594, 1068

Kohl, J. L., Noci, G., Cranmer, S. R., \& Raymond, J. C. 2006, A\&ARv, 13, 31

Kontar, E. P., Piana, M., Massone, A. M., Emslie, A. G., \& Brown, J. C. 2004, Sol. Phys., 225, 293

Kontar, E. P., Emslie, A. G., Piana, M., Massone, A. M., \& Brown, J. C. 2005, Sol. Phys., 226, 317

Kopp, R. A., \& Pneuman, G. W. 1976, Sol. Phys., 50, 85

Kozarev, K. A., Korreck, K. E., Lobzin, V. V., Weber, M. A., \& Schwadron, N. A. 2011, ApJ, 733, L25

Landi, E., Raymond, J. C., Miralles, M. P., \& Hara, H. 2010, ApJ, 711, 75

Landi, E., Del Zanna, G., Young, P. R., Dere, K. P., \& Mason, H. E. 2012a, ApJ, 744, 99

Landi, E., Raymond, J. C., Miralles, M. P., \& Hara, H. 2012b, ApJ, 751, 21

Lemen, J. R., Title, A. M., Akin, D. J., et al. 2012, Sol. Phys., 275, 17

Lin, J., \& Forbes, T. G. 2000, J. Geophys. Res., 105, 2375

Lin, J., Ko, Y.-K., Sui, L., et al. 2005, ApJ, 622, 1251

Liu, R., Liu, C., Wang, S., Deng, N., \& Wang, H. 2010, ApJ, 725, L84

Murphy, N. A., Sovinec, C. R., \& Cassak, P. A. 2010, J. Geophys. Res. (Space Phys.), 115, 9206

O’Dwyer, B., Del Zanna, G., Mason, H. E., Weber, M. A., \& Tripathi, D. 2010, A\&A, 521, A21

Patsourakos, S., Vourlidas, A., \& Stenborg, G. 2010, ApJ, 724, L188

Piana, M., Massone, A. M., Kontar, E. P., et al. 2003, ApJ, 595, L127
Plowman, J., Kankelborg, C., \& Martens, P. 2012, ApJ, submitted [arXiv: 1204.6306]

Prato, M., Piana, M., Brown, J. C., et al. 2006, Sol. Phys., 237, 61

Reeves, K. K., \& Golub, L. 2011, ApJ, 727, L52

Reeves, K. K., Linker, J. A., Mikić, Z., \& Forbes, T. G. 2010, ApJ, 721, 1547

Robbrecht, E., \& Wang, Y.-M. 2010, ApJ, 720, L88

Savage, S. L., Holman, G., Reeves, K. K., et al. 2012, ApJ, 754, 13

Schettino, G., Poletto, G., \& Romoli, M. 2010, ApJ, 708, 1135

Sterling, A. C., \& Hudson, H. S. 1997, ApJ, 491, L55

Sterling, A. C., \& Moore, R. L. 2005, ApJ, 630, 1148

Sturrock, P. A. 1966, Nature, 211, 695

Temmer, M., Veronig, A. M., Kontar, E. P., Krucker, S., \& Vršnak, B. 2010, ApJ, 712,1410

Temmer, M., Veronig, A. M., Vršnak, B., et al. 2008, ApJ, 673, L95

Testa, P., De Pontieu, B., Martínez-Sykora, J., Hansteen, V., \& Carlsson, M. 2012, ApJ, 758, 54

Tikhonov, A. N. 1963, Soviet Math. Dokl., 4, 1035

Török, T., \& Kliem, B. 2003, A\&A, 406, 1043

Vaiana, G. S., Krieger, A. S., \& Timothy, A. F. 1973, Sol. Phys., 32, 81

Vršnak, B., Sudar, D., \& Ruždjak, D. 2005, A\&A, 435, 1149

Vršnak, B., Poletto, G., Vujić, E., et al. 2009, A\&A, 499, 905

Webb, D. F., \& Howard, T. A. 2012, Liv. Rev. Sol. Phys., 9, 3

Weber, M. A., Deluca, E. E., Golub, L., \& Sette, A. L. 2004, in Multi-Wavelength Investigations of Solar Activity, eds. A. V. Stepanov, E. E. Benevolenskaya, \& A. G. Kosovichev, IAU Symp., 223, 321

Zhang, J., \& Dere, K. P. 2006, ApJ, 649, 1100

Zhang, J., Dere, K. P., Howard, R. A., Kundu, M. R., \& White, S. M. 2001, ApJ, 559,452 